\title{
MODELOS DE PREDICCIÓN Y CLASIFICACIÓN DE DEFICIENCIAS DE HIERRO EN HOJAS DE FRIJOL (Phaseolus vulgaris L.) CON REDES NEURONALES BAYESIANAS REGULARIZADAS Y ÁRBOLES DE CLASIFICACIÓN
}

\section{PREDICTION AND CLASSIFICATION MODELS OF IRON DEFICIENCIES IN COMMON BEAN (Phaseolus vulgaris L.) LEAVES USING BAYESIAN REGULARIZED NEURAL NETWORKS AND CLASSIFICATION TREES}

\author{
Edgar García-Cruz, Manuel Sandoval-Villa*, José A. Carrillo-Salazar, Paulino \\ Pérez-Rodríguez, Jorge D. Etchevers-Barra y Antonia Macedo-Cruz
}

Campus Montecillo, Colegio de Postgraduados. Km. 36.5 Carr. México-Texcoco. 56230, Montecillo, Estado de México, México.

*Autor para correspondencia (msandoval@colpos.mx)

\section{RESUMEN}

Los métodos de detección de deficiencias de hierro ( $\mathrm{Fe}$ ) en cultivos como el frijol (Phaseolus vulgaris L.) constituyen una herramienta valiosa en la toma de decisiones porque pueden utilizarse para predecir el estado nutrimental de las plantas en etapas tempranas. En esta investigación se usaron redes neuronales bayesianas regularizadas (BRNN, por sus siglas en inglés) y árboles de clasificación para llevar a cabo la predicción de dichas deficiencias basados en lecturas del SPAD 502, el cual se empleó para medir el índice de verdor de las hojas en el frijol. Se llevó a cabo un experimento con ocho tratamientos con diferentes variaciones en la concentración de $\mathrm{Fe}(0,20,40$, $60,80,100,150$ y $200 \%$ ) en la solución nutritiva. Durante 7 semanas se tomaron las mediciones promedio del índice de verdor de los tres foliolos de cinco repeticiones correspondientes a los ocho tratamientos y posteriormente los datos fueron utilizados para ajustar los modelos estadísticos antes mencionados. Con las BRNN, la correlación entre valores observados y predichos fue de 0.77 para el conjunto de datos en entrenamiento y de 0.54 0.71 para prueba. Para el caso de los árboles de clasificación, en etapa de entrenamiento el porcentaje de clasificaciones correctas fue $56.25 \%$ y disminuyó casi $30 \%$ cuando se llevó a cabo el procedimiento de validación. Por lo que para la presente investigación, el uso de BRNN constituye una herramienta valiosa para la predicción de deficiencias tempranas de Fe en el cultivo de frijol.

Palabras clave: SPAD, clorosis férrica, detección, nutrición, índice de verdor.

\section{SUMMARY}

The detection methods for iron (Fe) deficiencies in crops, such as common bean (Phaseolus vulgaris L.), are valuable decision-making tools that predict the nutritional status of plants in early stages. For this research, Bayesian regularized neural networks (BRNN) and classification trees were applied to predict iron deficiencies based on SPAD-502 readings that estimated the greenness index in common bean leaves. An experiment was carried out with eight treatments at different levels of Fe concentration in the nutrient solution $(0,20,40,60,80,100,150$ and $200 \%)$. For seven weeks, the average green index measurements of the three leaflets of five replicates corresponding to the eight treatments were taken, and the collected data were used to adjust the statistical models mentioned above. With BRNN, the correlation between observed and predicted values was 0.77 for the training data set and 0.54 0.71 for the test data. In the case of classification trees, in the training stage, the percentage of correct classifications was $56.25 \%$, and when the validation procedure was carried out, it decreased almost $30 \%$. Thus for this type of research, the use of BRNN constitutes a valuable tool for the prediction of early deficiencies of Fe in common bean crop.

Index words: SPAD, iron chlorosis, detection, nutrition, green index.

\section{INTRODUCCIÓN}

La deficiencia de hierro (Fe) es un problema mundial en la producción de cultivos en suelos calcáreos. Dentro de los cultivos afectados por la deficiencia de Fe, el frijol (Phaseolus vulgaris L.) es uno de ellos porque se encuentra dentro de la categoría de susceptible a la clorosis férrica (Hansen et al., 2007; Zaiter et al., 1992). De acuerdo con Clark (1991), este problema puede reducir hasta $100 \%$ del rendimiento del cultivo. La clorosis férrica se ve expresada en la planta una vez que el estado de la deficiencia es avanzado, y esta decoloración de las hojas se debe a un desequilibrio entre los contenidos de clorofila y los carotenos por la reducción del número de tilacoides y granas; de esta forma, hay una disminución de los pigmentos que recogen la luz: clorofilas a y $b$, y carotenos (Soldatini et al., 2000). A nivel molecular, el Fotosistema I (PSI) es el primer afectado por la deficiencia de Fe, por lo que disminuye el número de estos complejos, lo que resulta en un cuello de botella en el flujo de electrones necesarios en la fotosíntesis (Strzepek y Harrison, 2004; Yadavalli et al., 2012). Una vez que el daño a nivel molecular se ha dado, ha ocurrido ya una disminución al rendimiento del cultivo, por lo que las acciones correctivas que pudieran llevarse a cabo solo tendrían un efecto amortiguador. De aquí la importancia de llevar a cabo la detección a tiempo del problema para ejecutar acciones que puedan evitar un decremento en el rendimiento más allá de un umbral económico.

Uno de los métodos para realizar el diagnóstico nutrimental en el cultivo es el análisis químico en laboratorio; sin embargo, a pesar de la precisión que éstos puedan tener, su utilización constituye una inversión de tiempo que puede evitarse con métodos de detección in situ. En el caso de 
elementos como el nitrógeno $(\mathrm{N})$ existen aparatos específicos para la estimación del contenido de este elemento en el cultivo, no así para el caso de Fe. Un instrumento que permite estimar el contenido de $\mathrm{N}$ es el medidor de contenido de clorofila SPAD 502 (Konica Minolta Sensing, New Jersey, USA). Markwell et al. (1995) sugieren que probablemente la determinación de clorofila en la hoja por unidad de área es relativamente independiente de las especies, por lo que la relación de la clorofila con los valores SPAD sería la misma.

El análisis de $\mathrm{Fe}^{2+}$ en la hoja se ha llegado a tomar como un indicador del nivel de abastecimiento de hierro en la planta (Razeto y Valdés, 2006) e investigadores como Köseoğlua y Açikgöza (1995) han llevado a cabo experimentos donde se ha encontrado una alta correlación entre la concentración de clorofila y la de $\mathrm{Fe}^{2+}$ en la hoja de duraznero (Prunus persica L.), tanto en tejido fresco como en tejido seco. Lombard et al. (2010) han llevado a cabo trabajos con apoyo del SPAD 502 para la estimación de Fe en especies forestales como el álamo(Populus ssp.) y en árboles frutales como perales(Pyrus communis L.), en los que se usó este equipo para medir el contenido de clorofila (López-Millán et al., 2001). Se tomaron muestras de hojas con diferentes grados de deficiencias de Fe y donde se habían tomado previamente lecturas SPAD (Lombard et al., 2010). Posteriormente, la clorofila se extrajo con acetona al $100 \%$ y en presencia de ascorbato de sodio dado que se supone, existe correlación entre el contenido de Fe y de clorofila. De acuerdo con Lombard et al. (2010), el SPAD 502 , comparado con otros métodos de extracción caros o escalas de evaluación visual, este medidor de clorofila puede ser considerado como un indicador confiable de estimación del color, aunque cabe mencionar que no hay estudios hechos específicamente para correlacionar los valores de las lecturas SPAD con estatus nutrimental del Fe en el cultivo de frijol.

Existen diferentes modelos estadísticos que se han utilizado para relacionar las lecturas SPAD con la concentración de clorofila como análisis de regresión (Hirai et al., 2007; Lombard et al., 2010; Vesali et al., 2015). No obstante, una de las metodologías para el análisis de la información obtenida consiste en el uso de métodos estadísticos no paramétricos como las redes neuronales artificiales tal como se demuestra en el trabajo llevado a cabo por Vesali et al. (2015), quiénes sistematizaron los datos de valores SPAD, características de color e información obtenida de sensores de imagen para predecir la concentración de $\mathrm{N}$ en plantas de maíz (Zea mays L.) a través de una aplicación móvil y la cual aún no está disponible para uso comercial. El desempeño de los diferentes tipos de redes neuronales es variado; de acuerdo con MacKay (1992), éstas pueden usarse tanto para modelos de regresión como para cla- sificación. El mismo autor agrega que esta flexibilidad las hace capaces de descubrir relaciones más complejas en los datos que los modelos estadísticos tradicionales.

Por otro lado, la estadística Bayesiana provee un marco unificador para la generación de modelos, y evitar el problema de sobreajuste que generalmente presentan este tipo de modelos por el hecho de depender de un gran número de parámetros (Foresee y Hagan, 1997). Los modelos Bayesianos pueden ser utilizados con la finalidad de predecir la clase y clasificar cada caso no visto: un nuevo caso o ejemplo $j$, caracterizado sólo con los valores $x_{j}$ de las variables predictoras, se da una etiqueta clase según la categoría que tiene la máxima probabilidad a posteriori. Una propiedad útil de un clasificador bayesiano es que es óptimo, en el sentido que la tasa esperada de falsos positivos se reduce a un mínimo (Ripley, 1996), lo que constituye una ventaja adicional al problema del sobreajuste al ser un método supervisado. Correa et al. (2008) señalan que el objetivo de la clasificación supervisada es categorizar los casos $i$ dados por ciertas características $x_{i}=\left(x_{i}, \ldots, x_{i n}\right)^{\prime}$ en clases $r, c_{i,} i=7, \ldots, r, x_{i l}$ denota el valor de la variable $x_{1}$ observada en el caso $i$.

Aunque la aplicación de las redes neuronales bayesianas regularizadas (BRNN) no es nueva en agricultura, si lo es, el uso de métodos de aprendizaje de máquina en la predicción e Inteligencia Artificial, para predecir deficiencias de nutrientes. En un documento escrito por Bustos (2005), el autor hace un análisis de la inteligencia artificial en diferentes líneas de trabajo en la agricultura, lo que incluye el tipo de redes arriba mencionado. Dentro de algunos de los campos mencionados se encuentra la ingeniería postcosecha, el control ambiental, maquinaria y mecanización agrícola, ingeniería de riegos y producción vegetal, específicamente en aspectos nutricionales de los cultivos. De hecho, hay aplicaciones del uso de estas redes en investigaciones relacionadas con la categorización de estatus nutrimental de algún cultivo. Tal es el caso del trabajo llevado a cabo por Ma et al. (2014) en el que se discuten métodos de clasificación del verdor de los cítricos para categorizar en plantas sanas, infectadas por Huanglongbing (HLB), deficiencia de Fe y deficiencia de $\mathrm{N}$, mediante BRNN. Otra forma de categorización de la información es el uso de los árboles de clasificación porque proporcionan predictores bastante comprensibles en situaciones en las que hay variables que interactúan de forma complicada y de forma no lineal. La variable respuesta $Y$ es categórica, así, puede usarse información teórica para medir qué tanto puede aprenderse de ésta al saber el valor de otra variable discreta A. Hay dos tipos de predicciones que un árbol de clasificación puede hacer. El primer tipo es predicción puntual de la clase o categoría. El segundo tipo es la predicción de pertenencia a cada una de las clases (Shalizi, 2009). 
Para la presente investigación, y de acuerdo con la información anterior, el objetivo fue evaluar dos modelos de predicción del contenido de Fe en el cultivo de frijol, redes neuronales bayesianas regularizadas (BRNN) y árboles de clasificación por medio de lecturas SPAD a través del tiempo durante la etapa vegetativa de cultivo.

\section{MATERIALES Y MÉTODOS}

\section{Establecimiento del experimento, transplante y aplicación de tratamientos}

La siembra de semillas de frijol var. Negro Chapingo, se realizó bajo condiciones de invernadero en el Colegio de Postgraduados, en charolas de poliestireno y con perlita como sustrato durante el mes de junio de 2015. El riego se hizo diariamente con agua destilada.

Doce días después de la siembra (dds), éstas se trasplantaron bajo condiciones de hidroponía en vasos de poliestireno, y se continuó el riego con agua destilada. Posteriormente, la preparación de las soluciones nutritivas se llevó a cabo también con agua destilada y se tomó como base a la solución universal Steiner. Cada uno de los vasos en los que se trasplantó una plántula se tomó como unidad experimental. A partir de 80 unidades, se dividieron éstas en ocho grupos con 10 repeticiones. A cada grupo se le aplicó un tratamiento que correspondió a las variaciones de concentraciones de Fe. Los tratamientos fueron en condiciones de deficiencia (0,20,40,60 y $80 \%$ ) y exceso (150 y $200 \%$ ), además de la condición óptima o testigo (100 \%). Éstos se aplicaron 7 días después del trasplante (ddt). Durante 7 semanas se llevaron a cabo 20 mediciones del índice de verdor por cada tratamiento, se tomaron en cuenta cinco repeticiones por los ocho tratamientos, por medio de un SPAD 502 (Konica Minolta, New Jersey, USA), tales repeticiones correspondieron a cinco hojas, una hoja de cada planta, de las cuales se tomaron cinco lecturas por cada uno de los foliolos de la hoja para después obtener un promedio. Posteriormente, se tomó en cuenta el promedio de los tres foliolos. Los valores promedio por tratamiento fueron usados para generar el modelo (160 observaciones). Debe mencionarse que las mediciones se tomaron en la etapa vegetativa.

\section{Predicción de deficiencias de Fe mendiante redes neuronales bayesianas regularizadas (BRNN)}

Se usó un modelo de BRNN (Figura 1), entrenado a partir de un conjunto de datos con los que se pretende predecir la concentración de Fe en plantas de frijol (y), la que se considera como variable respuesta, que se supone es posible predecir utilizando dos covariables (variables de entrada): valores $\operatorname{SPAD}\left(x_{1}\right)$ y ddt $\left(x_{2}\right)$. Se parte de la utili- zación de $S$ neuronas de la capa interna, tres en este caso (Figura 1), las cuáles procesaron la información recibida de las variables de entrada para posteriormente generar una respuesta localmente por cada una de las neuronas. Los valores de entrada en cada una de las neuronas son combinados linealmente $\left(u_{1}, u_{2}, u_{3}\right)$ y la respuesta de esta operación es transformada de forma no lineal a través de una función de activación, la cual es una función que mapea las variables de entrada de la recta real al intervalo $(-1,1)$.

En este trabajo se empleó la función de activación tangente hiperbólica, $g(x)=2 / 1+\exp (-2 x)$ (Pérez-Rodríguez et al., 2012). Una vez que los datos fueron procesados en cada una de las neuronas $\left(g\left(u_{1}\right), g\left(u_{2}\right), g\left(u_{2}\right)\right)$, las salidas fueron combinadas linealmente, y se les asignó un peso específico a cada una de las neuronas para obtener la predicción de la variable respuesta. Los parámetros a estimar incluyeron; la media general $(\mu)$, los coeficientes de regresión en cada una de las neuronas: $\beta^{k}{ }_{{ }^{\prime}} \beta^{k}{ }_{1} \beta^{k}, k=7, \ldots, S$ (neuronas), los pesos de cada una de las neuronas: $W_{\gamma^{\prime}}, \ldots, w_{s^{\prime}}$ así como la varianza del error $\sigma^{2}$.

Para el ajuste del modelo, se utilizó el programa estadístico R (R Core Team, 2015), así como la biblioteca de funciones BRNN (Pérez-Rodríguez y Gianola, 2016). De acuerdo con estos autores, la biblioteca de funciones BRNN incluye una función con el mismo nombre que ajusta una red neuronal de dos capas como lo describen MacKay (1992) y Foresee y Hagan (1997). Además, usa el algoritmo de Nguyen y Widrow (1990) para asignar pesos iniciales y el algoritmo de Gauss-Newton para llevar a cabo la optimización.

La fase de entrenamiento de la BRNN puede describirse como un proceso de ajuste de los parámetros de forma gradual hasta que el vector de salida predicho aproxime lo más posible con el vector de salida deseado. El algoritmo utilizado para el ajuste del modelo es diferente al de retropropagación de los errores utilizado comúnmente en redes neuronales, en redes BRNN se minimiza una "suma de cuadrados penalizada". El algoritmo de entrenamiento se detiene una vez que la "suma de cuadrados penalizada" se estabiliza en tres evaluaciones sucesivas (Foresee y Hagan (1997) y Pérez-Rodríguez et al. (2012)). Una vez que los estimadores de los parámetros de interés han sido obtenidos, es posible evaluar si el número de nodos utilizados es suficiente para realizar la predicción sin realizar sobreajuste, porque es posible calcular fácilmente el número efectivo de parámetros; es decir, el número de parámetros que desde el punto de vista estadístico son diferentes de cero (Okut et al., 2011; Pérez-Rodríguez et al., 2012).

Posteriormente se determinó el poder predictivo del modelo mediante dos esquemas de validación cruzada: 


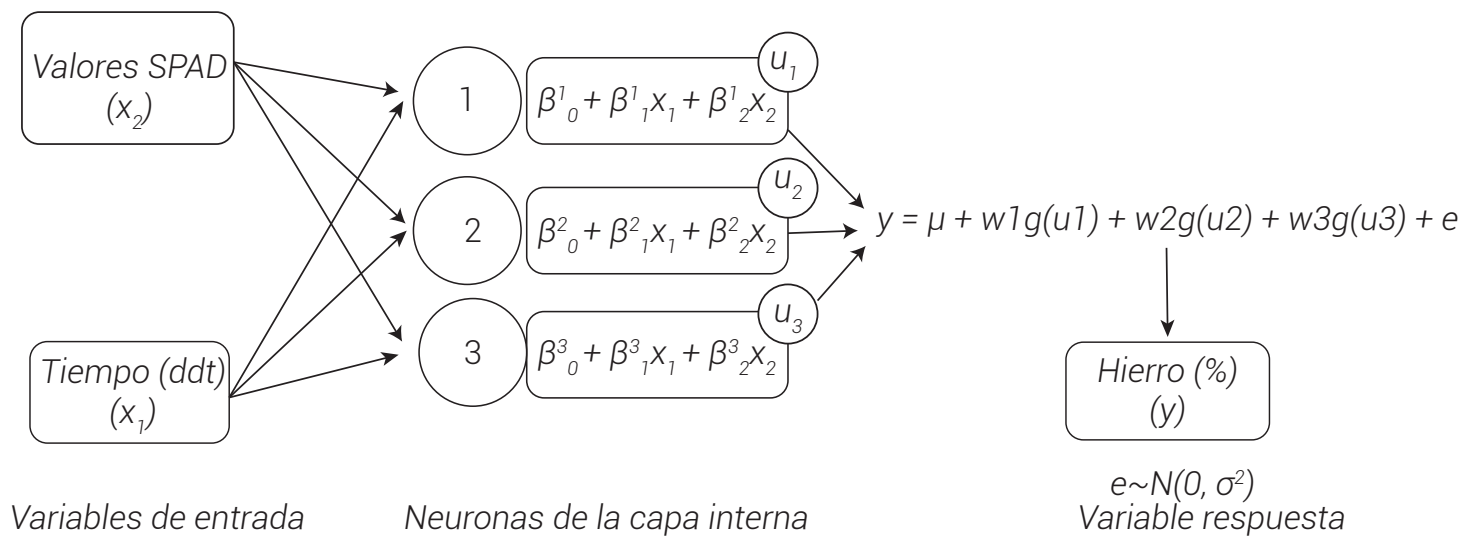

Figura 1. Representación gráfica del modelo de red neuronal utilizada para S: 3 neuronas.

1) Conjuntos aleatorios y 2) Conjuntos no aleatorios. En el primer caso, de acuerdo con James et al. (2013) el procedimiento involucra la división aleatoria de un conjunto de observaciones en un número $k$ de subconjuntos disjuntos (en este caso, ocho) de un tamaño igual aproximadamente. En un segundo procedimiento de validación no aleatorio, se tomaron también ocho grupos de acuerdo con las concentraciones de Fe (0,20,40,60, 80, 100 y 150). En cualquiera de los casos, el conjunto de datos es dividido en $k$ subconjuntos disjuntos $\left(S_{1}, \ldots, S_{8}\right)$. Cada uno de estos conjuntos puede utilizarse para medir el poder predictivo de los modelos. Por ejemplo, al utilizar el primer subconjunto, el conjunto de datos se divide en entrenamiento (los datos en $S_{1}, \ldots, S_{8}$ ) y prueba (los datos en $S_{1}$ ). Los modelos se ajustan a los datos en el conjunto de entrenamiento y luego se utiliza para predecir la variable respuesta para el conjunto de datos en prueba. Este procedimiento se repite $k$ número de veces (ocho en este caso) y al final del proceso se obtienen predicciones para los $k$ grupos, de donde se pueden obtener medidas que permiten evaluar el poder predictivo de los modelos, por ejemplo, correlaciones de Pearson entre valores observados y predichos o bien, tasas de aciertos y errores.

\section{Predicción de deficiencias de Fe usando árboles de clasificación}

De acuerdo con Loh (2011), los árboles de regresión, tanto como de clasificación son métodos de aprendizaje automático para la construcción de modelos a partir de datos. Los árboles de clasificación se construyen a partir de variables dependientes que tienen un número finito de valores sin ordenar y con un error de predicción medido en términos de clasificación errónea.

Por otro lado, para los árboles de predicción, la rutina utilizada fue tree en el programa estadístico R (Ripley, 2016). Estos modelos son obtenidos por la partición recursiva del espacio de datos y tras ajustar a un nivel de predicción simple dentro de cada partición. El mismo autor agrega que, en un problema de clasificación se tiene una muestra de entrenamiento con $n$ observaciones en una variable clase y que toma los valores $1,2, \ldots, k$, y $p$ variables de predicción, $x_{1}, x_{2}, \ldots, x_{p}$.

Para la presente investigación, la variable clase corresponde al porcentaje de Fe en la solución nutritiva (y), mientras que son dos las variables de predicción: ddt en que se tomó la medición con el SPAD $502\left(x_{1}\right)$, así como las lecturas promedio tomadas por cada hoja $\left(x_{2}\right)$. Para llevar a cabo la predicción se aplicó el siguiente algoritmo (Loh, 2011): 1) Comenzar en un nodo raíz; 2) Para cada variable $x$ ordenada, convertirla a una variable no ordenada $x^{\prime} y$ agrupar sus valores en el nodo en un pequeño número de intervalos. Si $x$ es no ordenada, fijar $x^{\prime}=x$; 3) Llevar a cabo una prueba de independencia chi cuadrada de cada variable $x^{\prime}$ contra $y$ en los datos en el nodo y calcular su significancia; 4) Elegir la variable $x \star$ asociada con la $x^{\prime}$ que tenga la probabilidad de significancia más pequeña; 5) Encontrar el conjunto $\left\{X^{*} \varepsilon S *\right\}$ que minimice la suma de los índices Gini (varianza binomial) y la use para dividir el nodo en dos hijos y donde s es un subconjunto de valores tomados por $x$; 6) Si hay convergencia, terminar. De otra forma, aplicar los pasos (2) al (5) para cada nodo hijo; y 7) Podar el árbol para encontrar el mejor tamaño del mismo.

\section{RESULTADOS Y DISCUSIÓN}

\section{Redes neuronales bayesianas regularizadas}

Se observó que las variables de entrada propuestas pueden predecir el estado nutrimental de Fe en el cultivo de frijol y el coeficiente de correlación de Pearson entre valores observados y predichos fue de 0.77 en el conjunto de datos en entrenamiento. En la Figura 2 puede apreciarse la tendencia de la concentración de Fe, la cual es similar 
al inicio de la toma de las lecturas. Como podría esperarse, los valores SPAD no aumentan mucho a medida que avanzan los días (17 a 25 ddt) en el caso del tratamiento con ausencia total de Fe, por lo que el índice de verdor es el más bajo con respecto al resto de los tratamientos. Adams et al. (2000) habían afirmado que las condiciones de deficiencia de Fe, entre otros elementos como el zinc (Zn) y manganeso $(\mathrm{Mn})$, causaban un decremento en la concentración de clorofila debido a que hay una relación directa con la ruta biosintética de ésta como elementos esenciales; Morales et al. (1991) describen que es en el transporte de electrones, en el proceso de la fotosíntesis, dónde la ausencia de Fe es causante de la clorosis.

Por el contrario, si se observa al testigo (Figura 2), puede notarse que tiene una tendencia a incrementar el índice de verdor, aunque no necesariamente se mantiene porque hay un decremento de los 30 a 40 ddt; además, el tratamiento con dosis de Fe, más allá del óptimo requerido, muestra un comportamiento en el que el índice de verdor es más alto entre los 25 a 30 ddt y posteriormente el decremento es pronunciado, para después registrar los valores SPAD más altos. Este resultado es similar a lo reportado por Kobraee et al. (2011) dónde concluyeron que los valores SPAD se incrementaron al aumentar la concentración de clorofila y cuando se aplicaron dosis más altas de Fe, entre otros micronutrimentos.

Deben también considerarse investigaciones anteriores, como la llevada a cabo por Papasavvas et al. (2008) en remolacha (Beta vulgaris L.), quiénes demostraron la correlación entre los valores SPAD y el contenido de clorofila, aunque enfocado a la concentración de $\mathrm{N}\left(\mathrm{NO}_{3}{ }^{-}\right)$en la planta. No obstante, debe tomarse en cuenta que tanto el $\mathrm{N}$ como el Fe están directamente relacionados con el verdor de las hojas, lo cual también se ha comprobado en trabajos de Ammari et al. (2009) en plantas de cítricos.

En validación cruzada, el coeficiente de correlación entre valores observados y predichos fue de 0.54 cuando la partición es no aleatoria. No obstante, cuando se lleva a cabo la validación cruzada con particiones aleatorias, el porcentaje de clasificaciones correctas resultó mayor (0.71). Esto se explica debido a que en los datos de entrenamiento se incluyó información de todos los grupos, incluido el que se estaba validando, no así en el caso anterior de validación cruzada no aleatoria. Cuando se llevó a cabo la validación cruzada con particiones aleatorias el procedimiento se repitió 1000 veces, y se predijeron aproximadamente $12 \%$ de las observaciones (20). En cada repetición se obtuvo la correlación entre valores observados y predichos y con dichas correlaciones, se obtuvo una distribución empírica de las mismas. La distribución de las correlaciones se muestra en la Figura 3. Mediante la distribución empírica de las correlaciones se obtuvo un intervalo de confianza al $95 \%$, de lo que se concluye que la correlación entre valores observados y predichos está entre 0.68 y 0.75 árboles de predicción y clasificación.

El árbol de clasificación obtenido se muestra en la Figura 4(a). El nodo principal es asignado a un valor de las lecturas SPAD (36.66), a partir del cual se bifurca en dos nodos

\section{Modelo ajustado en entrenamiento}

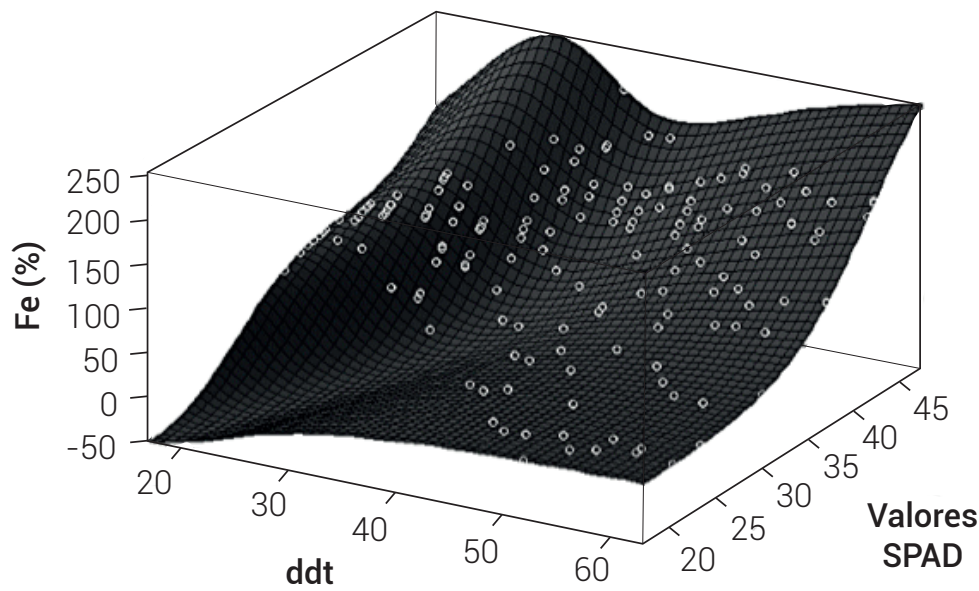

Figura 2. Representación gráfica tridimensional que muestra el comportamiento de las diferentes concentraciones de Fe en función del tiempo y el índice de verdor (valores SPAD) en el cultivo de frijol. Estos datos se usaron para el entrenamiento de la BRNN. Los puntos corresponden a las mediciones mientras que en la malla se reflejan los valores que corresponden a las predicciones obtenidas mediante la BRNN con S: 3 neuronas. 
A)

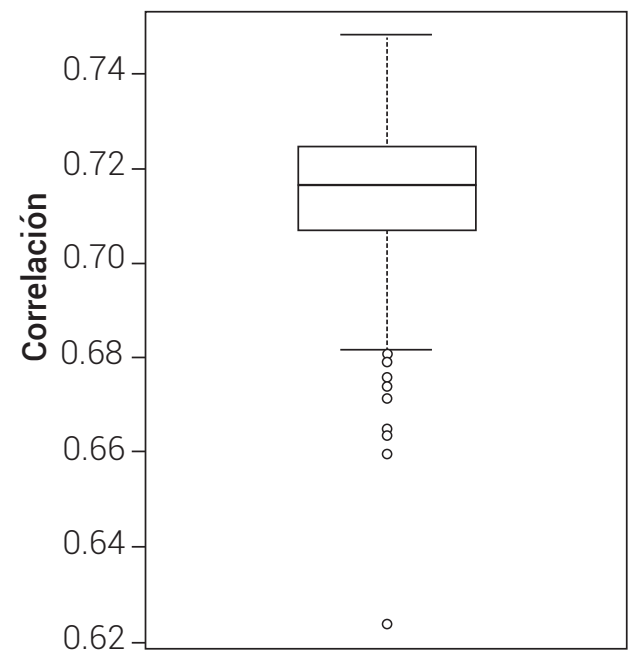

B)

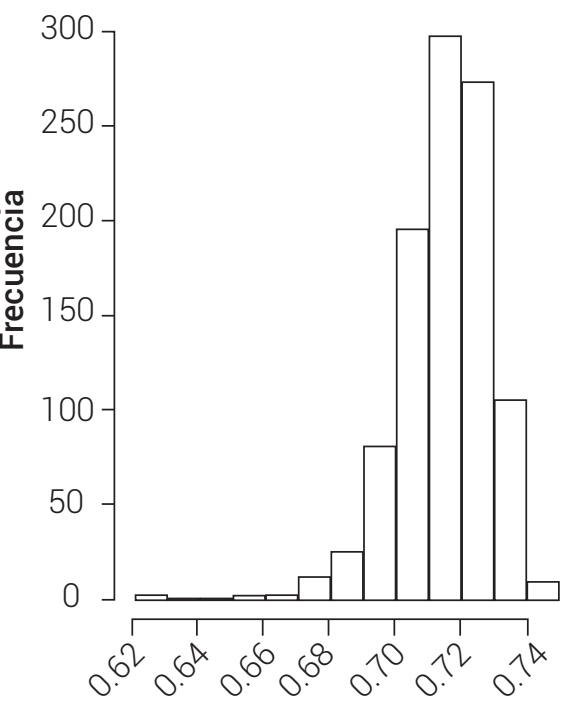

Correlación

Figura 3. Diagrama de cajas y bigotes para las correlaciones obtenidas mediante validación cruzada. Histograma de frecuencias de las diferentes correlaciones entre valores predichos contra valores observados obtenidas después de llevar a cabo el procedimiento de validación cruzada con ocho particiones aleatorias.

hijos, y está sujeto a si el valor SPAD es mayor o inferior al fijado al nodo principal. Por lo tanto, se observó que las concentraciones de $0 \%$ Fe siempre tuvieron valores SPAD inferiores a 34.83, excepto si el día que se tomó la lectura es posterior a los 37 ddt porque la concentración modelada será otra. Dicha concentración podrá ser 20, 40 o $60 \%$ si el valor SPAD es superior a 34.83, y depende del tiempo en que se haya tomado la lectura.

Concentraciones como 80, 100 y $150 \%$ de Fe siempre tendrían valores SPAD superiores a 36.66 , incluso, la concentración de $200 \%$ registraría valores superiores a 44.19 . Esta opción representa una alternativa sencilla para la discriminación entre diferentes clases de deficiencias debido a las condicionantes que presenta para descartar una $u$ otra opción, por lo que su aplicación en campo sería relativamente sencilla una vez que el método pudiera ser calibrado y mejorado.

Una forma más práctica de visualizar la información es a través de la Figura 3B. Por ejemplo, las concentraciones superiores (200 \%) a la óptima tendrán valores SPAD superiores a 44 a lo largo, de los 20 a los 60 ddt, aproximadamente, mientras que las concentraciones de $0 \% \mathrm{Fe}$ tendrán valores SPAD inferiores, de los 36 hasta los 32 ddt. A días posteriores, los valores SPAD inferiores a 36, pero superiores a 28, pertenecerán a la clase de 20 o $40 \%$ de Fe, y dependen de la ubicación en el tiempo en que se haya llevado a cabo la lectura. Dentro de los pocos trabajos similares con árboles de clasificación, Calderón et al.,
(2015) determinaron los parámetros de estrés que discriminarán entre clases de severidad de Verticillium. Uno de los parámetros usados fue la concentración de clorofila determinada por valores SPAD en cultivares de olivo (Olea europaea L.). Bajo estas consideraciones teóricas y dado que el hierro activo o soluble $\left(\mathrm{Fe}^{2+}\right)$ tiene una relación con el contenido de clorofila (Marsch et al., 1963), podría considerarse el SPAD 502 como una herramienta de detección del estatus nutrimental de Fe.

Como se observa en el Cuadro 1, la sensibilidad del modelo cambia de acuerdo con la concentración de Fe. El modelo tiene menos dificultad para asignar la clase al tratamiento con $0 \% \mathrm{Fe}$, mientras que los tratamientos con 60 y $150 \%$ son más difíciles de ubicar con apenas el $35 \%$ de clasificaciones correctas. En el primer caso, hay confusión de clases con el tratamiento más cercano, lo cual también puede observarse con los tratamientos de 40 y $200 \%$ Fe. Esto podría significar un inconveniente para el desempeño del modelo, aunque probablemente podrían desarrollarse rangos en los que pudiera ubicarse la deficiencia del Fe.

El promedio general de clasificaciones correctas del modelo para los datos completos, es de $56.2 \%$, por lo que se requiere de otras variables que puedan coadyuvar a discriminar entre las diferentes clases para mejorar los porcentajes de clasificaciones correctas. Se llevó a cabo el procedimiento de validación cruzada (Cuadro 2) con 1000 particiones aleatorias, del total de las observaciones, aproximadamente $88 \%$ de los datos se usó en entrenamiento, mientras que 
Valores SPAD $<36.655$

a)

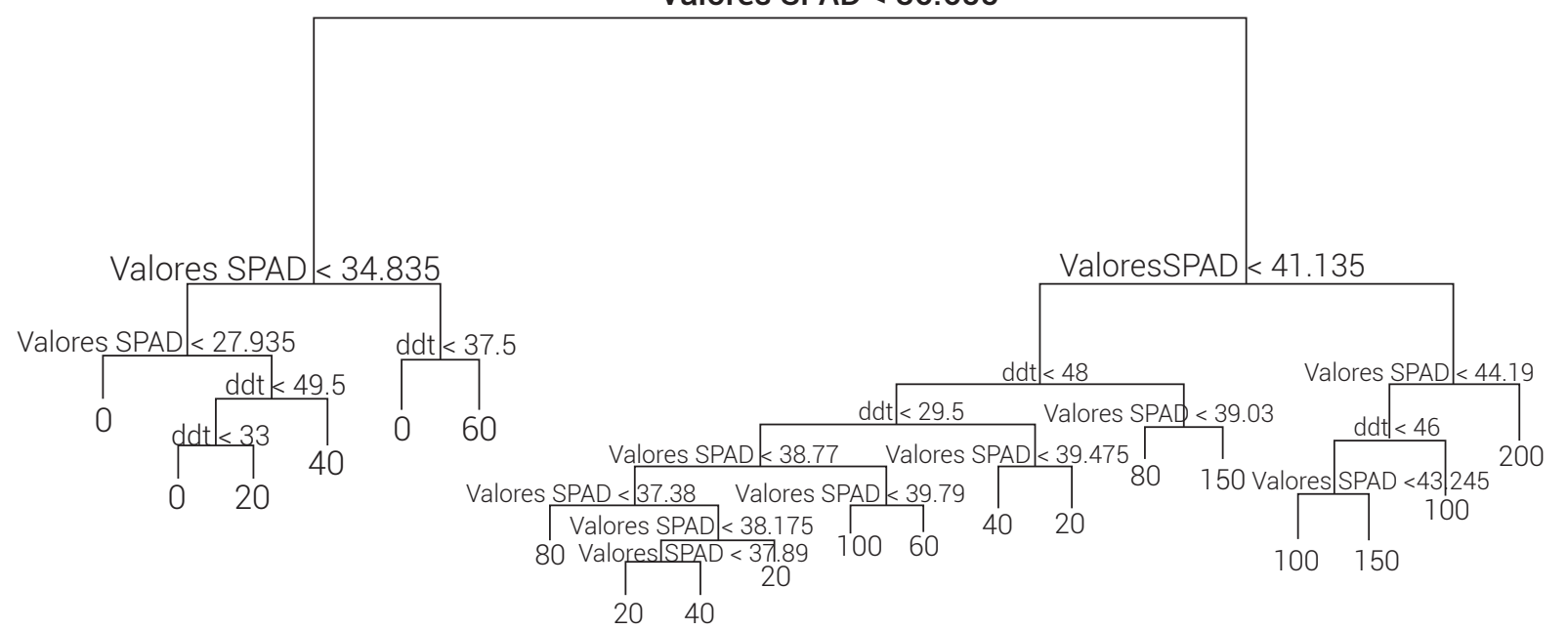

b)

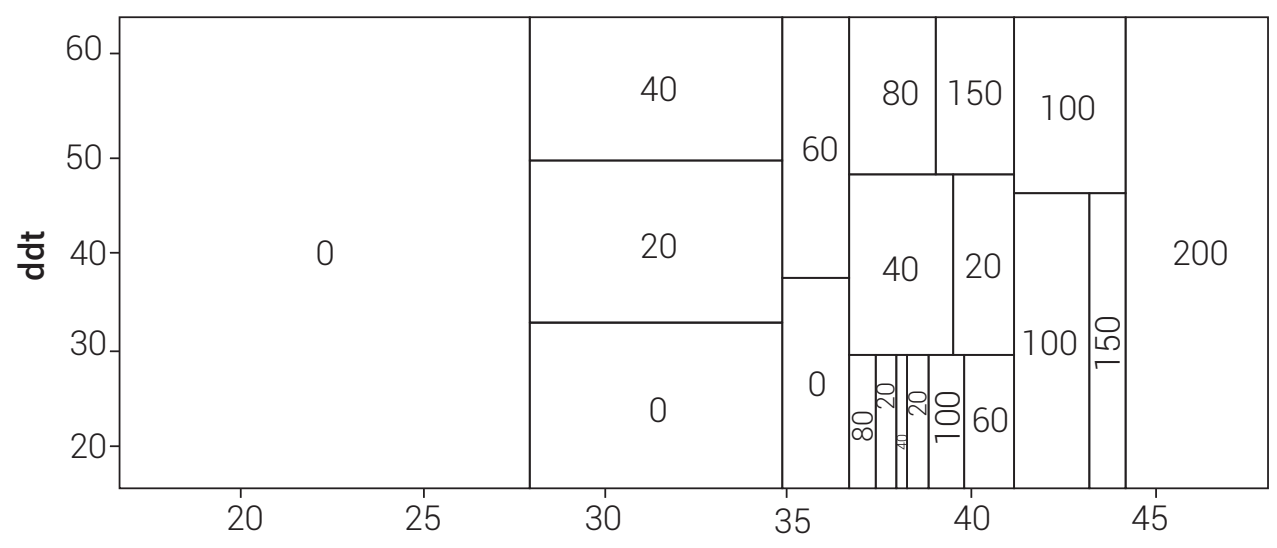

Figura 4. Predicción de Fe en el cultivo de frijol a partir del número de días después del transplante (ddt) y los valores SPAD mostrados en un (a) Diagrama de árbol y (b) Partición binaria del espacio.

Cuadro 1. Aciertos y errores de los valores observados contra valores modelados a través de árboles de clasificación para la medición de especificidad/sensibilidad del modelo en entrenamiento.

\begin{tabular}{crrrrrrrr}
\hline Clase & \multicolumn{7}{c}{ Clase predicha } \\
\cline { 2 - 8 } observada & 0 & 20 & 40 & 60 & 80 & 100 & 150 & 200 \\
\hline 0 & 95 & 0 & 5 & 0 & 0 & 0 & 0 & 0 \\
20 & 40 & $\mathbf{5 0}$ & 0 & 0 & 5 & 0 & 5 & 0 \\
40 & 5 & 10 & $\mathbf{5 5}$ & 20 & 0 & 5 & 5 & 0 \\
60 & 5 & 0 & 15 & $\mathbf{3 5}$ & 10 & 30 & 0 & 5 \\
80 & 0 & 15 & 5 & 5 & $\mathbf{4 5}$ & 15 & 15 & 0 \\
100 & 10 & 0 & 5 & 0 & 0 & $\mathbf{8 5}$ & 0 & 0 \\
150 & 5 & 10 & 0 & 5 & 5 & 25 & $\mathbf{3 5}$ & 15 \\
200 & 5 & 0 & 0 & 5 & 0 & 35 & 5 & $\mathbf{5 0}$ \\
Sensibilidad (\%) & 95 & 50 & 55 & 35 & 45 & 85 & 35 & 50 \\
\hline
\end{tabular}


Cuadro 2. Aciertos y errores para clase observada contra clase predicha a través de árboles de clasificación para la medición de especificidad/sensibilidad del modelo en prueba.

\begin{tabular}{|c|c|c|c|c|c|c|c|c|}
\hline \multirow{2}{*}{$\begin{array}{c}\text { Clase } \\
\text { observada }\end{array}$} & \multicolumn{8}{|c|}{ Clase predicha } \\
\hline & 0 & 20 & 40 & 60 & 80 & 100 & 150 & 200 \\
\hline 0 & 63.1 & 22.8 & 5.9 & 0.6 & 0.6 & 4.7 & 0.6 & 1.6 \\
\hline 20 & 39.1 & 17.5 & 19.9 & 3.4 & 8.9 & 5.1 & 4.4 & 1.7 \\
\hline 40 & 6.1 & 28.5 & 18.3 & 15.9 & 16.4 & 6.7 & 7.1 & 1.1 \\
\hline 60 & 4.9 & 3.3 & 20.2 & 15.1 & 20.0 & 23.8 & 6.1 & 6.6 \\
\hline 80 & 0.3 & 8.1 & 11.6 & 19.9 & 15.6 & 18.2 & 18.5 & 8.0 \\
\hline 100 & 8.7 & 4.0 & 4.6 & 18.2 & 8.7 & 37.2 & 7.3 & 11.4 \\
\hline 150 & 4.3 & 5.0 & 7.7 & 5.2 & 23.8 & 23.0 & 10.2 & 20.9 \\
\hline 200 & 3.5 & 2.3 & 0.6 & 5.5 & 10.0 & 26.3 & 18.9 & 32.9 \\
\hline Sensibilidad (\%) & 63.1 & 17.5 & 18.3 & 15.1 & 15.6 & 37.2 & 10.2 & 32.9 \\
\hline
\end{tabular}

el resto, $12 \%$ en promedio, se utilizó en validación; en cada réplica del proceso de validación se predicen aproximadamente 20 casos. A partir de estas últimas observaciones se generó el Cuadro 2, donde se muestra el porcentaje de clasificaciones correctas para cada una de las clases.

Del cuadro anterior, puede notarse que el porcentaje de clasificación para el caso de 0 \% Fe fue de $63 \%$, y corresponde a la clase a la que se le asignaron las observaciones de manera correcta. En cambio, clases como $150 \%$ Fe tuvieron bajos porcentajes de clasificaciones correctas, porque el algoritmo las ubicó en clases contiguas a la correcta, por lo que la sensibilidad del modelo no fue buena. Lo mismo ocurrió con clases como 20 \% Fe, donde la mayoría fue asignada a las clases contiguas. Probablemente, la explicación a la confusión pueda deberse más a causas fisiológicas del cultivo que a cuestiones estadísticas. En este trabajo solo se exploró la utilización de dos modelos estadísticos, sin embargo podrían probarse otros modelos como por ejemplo la regresión de mínimos cuadrados parciales. Lin y Huang (2011) en una investigación relacionada obtuvieron un coeficiente de correlación superior a 98 \% cuando se estimó el estatus del nitrógeno en hojas de colza (Brassica napus); no obstante, también se utilizaron la reflectancia espectral y espectroscopía de reflectancia del infrarrojo cercano como covariables.

\section{CONCLUSIONES}

Tanto los árboles de clasificación como las redes neuronales bayesianas regularizadas son herramientas útiles en el análisis de la información que no se ajusta a modelos lineales, ya sea para variables que puedan ser caracterizadas como cualitativas o cuantitativas; sin embargo, con la adición de covariables podría robustecerse la modela- ción de la información para obtener mejores resultados en etapa de prueba del modelo. En el primer caso de árboles de clasificación, el porcentaje global de clasificaciones correctas es de $44 \%$, mientras que en el caso de las BRNN, el coeficiente de correlación de Pearson entre valores observados y predichos puede alcanzar un valor de 0.71 . No obstante, la predicción de las deficiencias de Fe en el cultivo de frijol es posible mediante valores SPAD y su distribución en el tiempo (fenología del cultivo).

\section{BIBLIOGRAFÍA}

Adams M. L., W. A. Norvell, W. D. Philpot and J. H. Peverly (2000) Spectral detection of micronutrient deficiency in 'Bragg' soybean. Agronomy Journal 92:261-268.

Ammari T. G., A. B. Tahboub and T. R. Abu-Zahra (2009) Management of Iron deficiency stress in citrus through soil application of vivianite to a calcareous soil. International Journal of Botany 5:186-189.

Bustos M. J. R. (2005) Inteligencia artificial en el sector agropecuario. Reporte técnico. In: Seminario de Investigación I: 2005-II. 299622. Universidad Nacional de Colombia, Ingeniería de Sistemas y Computación. 8 p.

Calderón R., J. A. Navas-Cortés and P. J. Zarco-Tejeda (2015) Early detection and quantification of verticillium wilt in olive using hyperspectral and thermal imagery over large areas. Remote Sensing 7:5584-5610

Clark R. B. (1991) Iron: unlocking agronomic potential. Solutions 35:2428.

Correa M., C. Bielza, J. Pamies-Teixeira y J. R. Alique (2008) Redes Bayesianas vs. Redes Neuronales en Modelos para la Predicción del Acabado Superficial. XVII Congreso de Máquinas-Herramienta y Tecnologías de Fabricación. San Sebastián, España. 14 p.

Foresee F. D. and M. T. Hagan (1997) Gauss-Newton approximation to Bayesian regularization. Proceedings of the 1997 International Joint Conference on Neural Networks. The International Neural Networks Society. Houston, Texas. pp:1930-1935.

Hansen N. C., B. G. Hopkins, J. W. Ellsworth and V. D. Jolley (2007) Iron nutrition in field crops. In: Iron Nutrition in Plants and Rhizospheric Microorganisms. L. L. Barton and J. Abadia (eds.). Springer Netherlands. Dordrecht, The Netherlands. pp:23-59.

Hirai M., K. Higuchi, H. Sasaki, T. Suzuki, T. Maruyama, M. Yoshiba and T. Tadano (2007) Contribution of iron associated with high-molecular-weight substances to the maintenance of the SPAD value of young leaves of barley under iron-deficient conditions. Soil Science and Plant Nutrition 53:612-620 
James G., D. Witten, T. Hastie and R. Tibshirani (2013) An Introduction to Statistical Learning with Applications in R. Springer-Verlag. New York, USA. 426 p.

Kobraee S., K. Shamsi and S. Ekhtiari (2011) Soybean nodulation and chlorophyll concentration (SPAD value) affected by some of micronutrients. Annals of Biological Research 2:414-422.

Köseoğlua A. T. and V. Açikgöza (1995) Determination of iron chlorosis with extractable iron analysis in peach leaves. Journal of Plant Nutrition 18:153-161.

Lin S. and X. Huang (2011) Advances in Computer Science, Environment, Ecoinformatics, and Education. International Conference, CSEE 2011. Wuhan, China. Proceedings, Part III. Springer Berlin Heidelberg. Heidelberg, Germany. 634 p.

Loh W. Y. (2011) Classification and regression trees. Wiley Interdisciplinary Reviews: Data Mining and Knowledge Discovery 1:14-23.

Lombard K., M. O'Neill, J. Mexal, A. Ulery, B. Onken, G. Bettmann and R. Heyduck (2010) Can soil plant analysis development values predict chlorophyll and total Fe in hybrid poplar? Agroforestry Systems 78:1-11

López-Millán A. F., F. Morales, A. Abadía and J. Abadía (2001) Iron deficiency-associated changes in the composition of the leaf apoplastic fluid from field-grown pear (Pyrus communis L.) trees. Journal of Experimental Botany 52:1489-1498.

Ma H., H. Y. Ji and S. L. Won (2014) Detection of citrus greening based on Vis-NIR spectroscopy and spectral feature analysis. Guang Pu Xue Yu Guang Pu Fen Xi 34:2713-2718.

MacKay D. J. C. (1992) Bayesian interpolation. Neural Computation 4:415-447.

Markwell J., J. C. Osterman and J. L. Mitchell (1995) Calibration of the Minolta SPAD-502 leaf chlorophyll meter. Photosynthesis Research 46:467-472.

Marsh H. V. Jr., H. J. Evans and G. Matrone (1963) Investigations on the role of iron in chlorophyll metabolism I. Effect of iron deficiency on chlorophyll and heme content and on the activities of certain enzymes in leaves. Plant Physiology 38:632-638.

Morales F., A. Abadía and J. Abadía (1991) Chlorophyll fluorescence and photon yield of oxygen evolution in iron-deficient sugar beet (Beta vulgaris L.) leaves. Plant Physiology 97:886-893.

Nguyen D. and B. Widrow (1990) Improving the learning speed of 2-layer neural networks by choosing initial values of the adaptive weights. In: Proceedings of the 1990 International Joint Conference on Neural Networks. 17-21 June. San Diego, California. 3:21-26.

Okut H., D. Gianola, G. J. M. Rosa and K. A. Weigel (2011) Prediction of body mass index in mice using dense molecular markers and a regu- larized neural network. Genetics Research 93:189-201.

Papasavvas A., V. Triantafyllidis, G. Zervoudakis, G. Kapotis, Y. Samaras and G. Salahas (2008) Correlation of SPAD-502 meter readings with physiological parameters and leaf nitrate content in Beta vulgaris. Journal of Environmental Protection and Ecology 9:43-48

Pérez-Rodríguez P., D. Gianola, K. A. Weigel, G. J. Rosa and J. Crossa (2012) Technical note: An R package for fitting Bayesian regularized neural networks with applications in animal breeding. Journal of Animal Science 91:3522-3531.

Pérez R. P. and D. Gianola (2016) BRNN: Bayesian regularization for feed-forward neural networks. R package version 0.6. https:// CRAN.R-project.org/package=brnn. (Febrero 2017).

R Core Team (2015) R: a language and environment for statistical computing. R Foundation for Statistical Computing, Vienna, Austria 3518 p. https://cran.r-project.org/doc/manuals/r-release/fullrefman.pdf. (Febrero 2017)

Razeto B. y G. Valdés (2006) Análisis de hierro soluble en tejidos para diagnosticar el déficit de hierro en nectarino. Agricultura Técnica 66:216-220.

Ripley B. D. (1996) Pattern Recognition and Neural Networks. Cambridge University Press. Cambridge, U.K. 403 p.

Ripley B. (2016) Tree: Classification and regression trees. R package version 1.0-37. https://CRAN.R-project.org/package=tree. (Febrero 2017)

Shalizi C. (2009) Classification and Regression Trees. Data Mining. Carnegie Mellon University. Pittsburgh, PA. 25 p.

Soldatini G. F., M. Tognini, A. Castagna, B. Baldan and A. Ranieri (2000) Alterations in thylakoid membrane composition induced by iron starvation in sunflower plants. Journal of Plant Nutrition 23:1717-1732

Strzepek R. F. and P. J. Harrison (2004) Photosynthetic architecture differs in coastal and oceanic diatoms. Nature 431:689-692.

Vesali F., M. Omid, A. Kaleita and H. Mobli (2015) Development of an android app to estimate chlorophyll content of corn leaves based on contact imaging. Computers and Electronics in Agriculture 116:211-220

Yadavalli V., C. C. Jolley, C. Malleda, B. Thangaraj, P. Fromme and R. Subramanyam (2012) Alteration of proteins and pigments influence the function of photosystem I under iron deficiency from Chlamydomonas reinhardtii. PLoS ONE 7:e35084. http://dx.doi org/10.1371/journal.pone.0035084

Zaiter H. Z., D. P. Coyne, R. B. Clark, D. T. Lindgren, P. T. Nordquist, W. W. Stroup and L. A. Pavlish (1992) Leaf chlorosis and seed yield of dry beans grown on high-pH calcareous soil following foliar iron sprays. HortScience 27:983-985. 